\title{
Usefulness of Bacteriological Monitoring of Endoscope Reprocessing
}

\author{
Julia Kovaleva and Astrid Buss \\ University Medical Center Groningen, Laboratory for Infectious Diseases Groningen, \\ The Netherlands
}

\section{Introduction}

Flexible endoscopy is a widely used diagnostic and therapeutic procedure. In The Netherlands, a 26\% increase in the number of endoscopies from 325.000 in 1999 to almost 410.000 in 2004 was found. The total number of endoscopic retrograde cholangiopancreatographies (ERCP) was estimated at 14.596 in 2004 (Terhaar sive Droste et al., 2006).

Contaminated endoscopes have been linked to more outbreaks of healthcare-associated infections than any other medical device. During the period 1974-2004, 70 outbreaks following endoscopy procedures were reported in the USA in 64 scientific articles (SeoaneVazquez et al., 2006). A total of 10637 patients were exposed to contamination agents during 28 outbreaks. Inadequate decontamination procedures were the leading cause of contamination; equipment malfunction became the second cause of contamination during the period 1990-2004. More than 91\% of the infections identified could be prevented if quality control systems are improved. However, the true transmission during endoscopy may go unrecognized because of inadequate surveillance or no surveillance at all, low frequency and absence of clinical symptoms (Srinivasan, 2003).

Endoscopes become heavily contaminated with blood, secretions and microorganisms during use, and because each endoscope may be used for different patients in a single day, it is essential to clean and disinfect them effectively between every endoscopic procedure in patients. Accurate reprocessing of flexible endoscopes must involve cleaning, followed by high level disinfection with further rinsing and drying before storage. Most contemporary flexible endoscopes cannot be heat-sterilized and are designed with multiple channels and ports which are exposed to body fluids and are difficult to clean and disinfect (Nelson et al., 2001). Growth of biofilms inside endoscope channels can result in failure of the endoscope reprocessing and is an important factor in the pathogenesis of endoscopy-related infections (Buss et al., 2008; Kovaleva et al., 2009). Such events should be prevented by well instructed personnel, well functioning washing, disinfection and drying equipment and observance of general hygiene guidelines in the endoscopy center.

Bacterial transmission from endoscopes to patients tends to be incidentally identified when an unusual species or a microorganism with an uncommon resistance profile is involved. The microorganisms most frequently associated with iatrogenic transmission during endoscopy are gram-negative bacteria (Pseudomonas aeruginosa, Serratia marcescens and Salmonella species), mycobacteria and yeasts (Buss et al., 2008; Kovaleva et al., 2009; Spach et 
al., 1993). These microorganisms can be transferred from previous patients or contaminated cleaning sources (reprocessing equipment, water supply lines) by contaminated endoscopes or accessories (Srinivasan, 2003).

Endoscopy-related outbreaks are associated with high costs including the costs of the epidemiological investigation of the outbreak, the diagnosis and treatment of the affected patients, and the use of resources cost associated with endoscope unit inactivity (SeoaneVazquez et al., 2006).

Microbiological surveillance of endoscope reprocessing is appropriate to trace contamination of endoscopes and to prevent contamination and infection in patients after endoscopic procedures. Routine microbiological monitoring of endoscope reprocessing has been recommended by several organizations (Beilenhoff et al., 2008; Systchenko et al., 2000). The repeated detection of an infection with the same microorganism in patients after endoscopic procedures in the University Medical Center Groningen (UMCG), The Netherlands, led to the implementation of the surveillance system for evaluation of the efficacy of the endoscope reprocessing (Buss et al., 2008; Kovaleva et al., 2009).

\section{Endoscopy-related infections}

Flexible endoscopes belong to semi-critical devices which come in contact with mucus membranes or non intact skin during endoscopic procedures. Endoscopes for therapeutic procedures (bronchoscopy, ERCP) are used in sterile body cavities. They are frequently designed with small lumina and multiple channels which are difficult to clean and disinfect. Such endoscopes should be sterilized or receive an intensive disinfection procedure (Alvarado \& Reichelderfer, 2000).

Infections related to endoscopes can be divided into two types: endogenous and exogenous. Endoscopic procedures most often result in endogenous infections (i.e., infections resulting from the patient's own microbial flora) and Escherichia coli, Klebsiella, Enterobacter species and enterococci are generally isolated (Spach et al., 1993). Endogenous infections are associated with endoscopy but can not be prevented by well controlled disinfection procedures. The exogenous microorganisms most frequently associated with transmission during bronchoscopy are P. aeruginosa and Mycobacterium tuberculosis, atypical Mycobacterium species, and $P$. aeruginosa the most common in gastrointestinal endoscopy (Nelson \& Muscarella, 2006). These microorganisms can be transmitted from previous patients or contaminated reprocessing equipment by contaminated endoscopes or its accessory equipment. Exogenous infection should be prevented by strict endoscope disinfection procedures (Srinivasan, 2003).

\subsection{Endoscopy-related infection associated with gastrointestinal endoscopy}

Bacteraemia occurs in any situation where there is damage of the mucosal membrane (for example, during teeth cleaning). The presence of bacteraemia after an endoscopic procedure does not indicate that serious clinical infection is present. Microscopic tissue trauma occurring during endoscope insertion can result in the passage of microflora into the bloodstream. The microorganisms isolated from the blood cultures belong to the oropharyngeal commensal microflora and are general of low pathogenicity (O'Connor et al., 1983).

The important risk factors of endoscopy-related infections in gastrointestinal endoscopy are the number of microorganisms present inside the endoscope or its accessories or the growth 
of a biofilm, invasive endoscopic procedures resulting in tissue damage, compromised immune status of the patient and the presence of infective foci (abscess, cholangitis) during an endoscopic procedure (Gastroenterological Society of Australia, 2006).

The role of antibiotic prophylaxis in gastrointestinal endoscopy is controversial. Prophylactic antibiotic administration is not recommended for any gastrointestinal endoscopic procedure (Hirota et al., 2003). Only patients with bile duct obstruction, immunosuppression or previous endocarditis may need the prophylaxis during ERCP or other therapeutic gastrointestinal endoscopic procedure (Cotton et al., 2008). Recent studies examining antibiotic prophylaxis prior to ERCP concluded that it may reduce the incidence of bacteraemia, but did not reduce the incidence of clinical sepsis or cholangitis. Optimum benefit of antibiotics is obtained if therapeutic levels are present in the tissues at the time of endoscopic procedure. Antibiotic prophylaxis should be started intravenously at least one to two hours before the procedure (Cotton et al., 2008; Harris et al., 1999; Gastroenterological Society of Australia, 2006).

Despite the large number of annually performed procedures, iatrogenic infections due to flexible gastrointestinal endoscopy are apparently rare. ERCP is the only endoscopic procedure which has been associated with a significant rate of procedure-induced infection (Christensen et al., 2004). The common pathogenic microorganisms encountered in the biliary tree are P. aeruginosa, E. coli, Klebsiella, Bacteroides species and enterococci.

A complication of ERCP can be defined as any event occurring during the 30-day period after the procedure that changed the health status of a patient negatively. Post-ERCP complications can be divided into mild (required up to 3 days in hospital), moderate (required 4 to 10 days of hospitalization) and severe complications (required more than 10 days of hospitalization, surgical intervention and/or resulted in the death of the patient) (Cotton et al., 1991).

Cholangitis and bacteraemia following ERCP can occur due to exogenous source (contaminated endoscope or accessories) or endogenous bacterial flora. Bloodstream infection is one of the most serious complications of this procedure, with the actual incidence varied from $0 \%$ to $27 \%$ in different populations and with the $8 \%-20 \%$ mortality rate (Anderson et al., 2008; Kullman et al., 1992). Cholangitis, the most frequent infective complication of ERCP, most commonly results from inadequate drainage of infected and obstructive biliary duct system (Low et al., 1980). Post-ERCP cholangitis is defined by a typical clinical picture (temperature $>38^{\circ} \mathrm{C}$, upper abdominal colicky pain, cholestasis/jaundice) without evidence of other concomitant infections with/without positive gall cultures obtained during the biliary drainage (Masci et al., 2001).

\subsubsection{Role of Pseudomonas aeruginosa in endoscopy-related infections}

$P$. aeruginosa, a gram-negative opportunistic pathogen, is the most commonly reported organism responsible for transmission of infection during gastrointestinal endoscopy. It is known by its preference for a moist environment (wet endoscope channels after reprocessing, hospital water supply) (Nelson \& Muscarella, 2006). Pseudomonas is well known to be able to form biofilms, and these biofilms are extremely difficult to remove from plumbing, automatic endoscope reprocessors and endoscope channels (Pajkos et al., 2004).

Pseudomonas contamination of endoscopes was first reported in the mid-70s (Bilbao et al., 1976) and is adequately documented as a serious clinical cause of ERCP-associated infection by 1980 (Low et al., 1980). Outbreaks of P. aeruginosa sepsis and cholangitis following ERCP 
continue to be reported. Frequently, the infection has not been recognized because of inadequate surveillance, low frequency and absence of clinical symptoms. It comes to light only as a result of infection control procedures.

According to the recent reports, $P$. aeruginosa transmission during gastrointestinal endoscopy has been attributed to (1) inadequate high-level disinfection of the endoscope channels after endoscope reprocessing prior to storage (Allen et al., 1987; Fraser et al., 2004), (2) colonization of the water supply to the endoscope (Bass et al., 1990) and (3) defective disinfecting machines, failure in design or defects in endoscope channels and accessories (Alvarado et al., 1991; Nelson \& Muscarella, 2006). Serotype 10 of P. aeruginosa was predominating in the published reports of Pseudomonas transmission (Allen et al., 1987; Alvarado et al., 1991). This serotype persisted in the outbreaks despite the intensive disinfection procedures. This phenomenon was linked to biofilm formation but no explanation was found as to why this serotype was isolated.

Inadequate drying of the endoscope channels prior to storage was responsible for the outbreaks of Pseudomonas bacteraemia after ERCP (Allen et al., 1987; Classen et al., 1988). P. aeruginosa remaining in the endoscope moist channels after reprocessing can colonize and multiply to high numbers during storage. This situation can result in an increased risk of transmission to the first patient of the day during an ERCP procedure.

\subsubsection{Example of an outbreak of Pseudomonas sepsis after ERCP}

We have recently been confronted with an outbreak of multidrug-resistant $P$. aeruginosa sepsis affecting six patients after ERCP. Our registration system enabled us to retrieve one of three endoscopes daily in use as the possible source of infection. Epidemiologic investigation including microbiological sampling of the implicated endoscope, the washer disinfectors and the tubes connecting the washer disinfectors and the endoscopes was performed. The results of recent surveillance cultures from diagnostic and therapeutic endoscopes were reviewed. Environmental investigations to determine potential reservoirs or sources of contamination included wash tables, sinks, drying cabinets, hand soap and detergent soap for endoscopes. The medical records of all patients who underwent ERCP with this endoscope from introduction in service until it was removed from service were reviewed.

All of the cultures from the washer disinfectors, the connecting tubes, other endoscopes, and from the environmental surfaces in the endoscopy center were negative for P. aeruginosa. Epidemiologic investigation found that the implicated ERCP endoscope had contamination with $P$. aeruginosa in microbiological surveillances before the outbreak, despite intensive high level disinfection procedures. The endoscope was removed from service and underwent sterilization with ethylene oxide. Following gas sterilization, the implicated endoscope was found to have P. aeruginosa-negative cultures and was re-introduced into service. However, 4 months later, it was again contaminated with $P$. aeruginosa. The endoscope was sent to the manufacturer for repair. Structures suggesting the presence of biofilm were found on the inner surface of the undamaged endoscope channels. The endoscope channels were changed.

The available $P$. aeruginosa strains were subjected to molecular typing by repetitive DNA sequence-based polymerase chain reaction (rep-PCR) using the DiversiLab System for DNA fingerprinting (BioMerieux, France). The isolates from the six patients and from the endoscope channels, obtained before gas sterilization, showed matching patterns (similarity above 95\%) (Kovaleva et al., 2009). 


\subsection{Endoscopy-related infection associated with bronchoscopy}

During the period 1974-2004, bronchoscopy procedures accounted for $47.5 \%$ of the endoscopy-related infections in the USA and $21.1 \%$ of the infections in other countries (Seoane-Vazquez et al., 2007). Several studies have found bronchoscopy procedures to be relatively more prone to infection transmission than gastrointestinal endoscopic procedures (Seoane-Vazquez et al., 2007; Spach et al., 1993).

The microorganisms most frequently associated with transmission during bronchoscopy are gram-negative bacilli, particularly $P$. aeruginosa or Enterobacteriaceae or infection with $M$. tuberculosis or other mycobacteria (Cêtre et al., 2005; Nelson \& Muscarella, 2006). While isolation of various Enterobacteriaceae from the lungs is not rare, severe nosocomial pneumonia is not frequently diagnosed in patients during infection outbreaks. It is possible that the incidence of pneumonia related to bronchoscopy is underestimated (Shimonom et al., 2008). The pathogens of nosocomial pneumonia are related to the timing of onset. Microorganisms responsible for early-onset nosocomial pneumonia are generally endogenous. In late-onset nosocomial pneumonia, the causative microorganisms include potentially multidrug-resistant nosocomial organisms such as $P$. aeruginosa.

Three large outbreaks of $P$. aeruginosa infections following flexible bronchoscopy associated with a loose port of the biopsy channel of the bronchoscope were reported in the literature (Cêtre et al., 2005; Kirschke et al., 2003; Srinivasan et al., 2003). Another two Pseudomonas outbreaks were associated with a damaged internal channel caused by defective biopsy forceps (Corne et al., 2005). A contaminated bronchoscope washer disinfector was the source of the several Pseudomonas outbreaks (Schelenz \& French, 2000; Shimonom et al., 2008).

Numerous reports of mycobacterial transmission by flexible bronchoscopy have been reported. Mycobacterial infections during bronchoscopy have been related to contaminated suction valves (Wheeler et al., 1989), cracked biopsy channels (Pappas et al., 1982), contaminated topical anaesthetic solutions (Leers et al., 1980) and contaminated disinfecting machines (Bryce et al., 1993; Fraser et al., 1992).

The last two years we were confronted with repeated contaminations of flexible bronchoscopes with fastidiously growing Methylobacterium. Methylobacterium was also isolated from the broncho-alveolair lavages of the patients after bronchoscopy with the contaminated bronchoscopes. We consider Methylobacterium as a contaminant being the cause of pseudo-infections because no patient manifested true infection with this bacterium. Methylobacterium is a fastidious, slowly growing gram-negative rod that forms pink colonies on agar plates and is a common contaminant in water (Sanders et al., 2000). Nevertheless it has been described as opportunistic human pathogen and has been shown to be able to cause colonization and infections in immunocompromised patients (Kaye et al., 1992; Liu et al., 1997). Two publications of Methylobacterium contamination related to endoscopic procedures were found in the literature. One patient developed Methylobacterium bacteraemia following ERCP with the contaminated endoscope (Imbert et al., 2005). A pseudo-outbreak of Methylobacterium mesophilicum and Mycobacterium chelonae was described in patients who underwent bronchoscopy. M. mesophilicum was isolated from bronchoalveolar lavages obtained from ventilated patients. Methylobacterium grew from the bronchoscopes and automated washers which were revealed as the source of the contamination. The presence of biofilm was demonstrated on tubing from the manifold block to the top of the reservoir (Kressel \& Kid, 2001). 


\section{Microbiological surveillance of endoscope reprocessing}

Endoscopes, especially the instruments with highly refined technical properties, can be cleaned and disinfected but not sterilized after use. This implies the risk of settlement of biofilm producing species, which can not be removed with current cleaning and disinfection procedures. A currently applied surveillance protocol for endoscopes demonstrates recurrent colonization of these instruments. Occasionally this leads to series of infections in patients, who become severely ill with pathogenic species being part of the biofilm which has settled in the interior of the endoscope.

In The Netherlands the current guidelines advise no routine microbiological surveillance of endoscopes, only under special circumstances like a defect of the endoscope or in the disinfection process and also in case of an outbreak (Dutch Infection Prevention Working Party, 2004). In our opinion is a good working disinfection process no guarantee on "clean" endoscopes, because damage of the endoscope itself occurs during use, e.g. due to through scratches in the working channel by the biopsy forceps, and forms a niche for rising microbiological biofilm. Outbreaks can easily be overlooked and are often only recognized if special microorganisms are involved, like outbreaks caused by a multiresistant $E$. coli in 2002 and by a multi-resistant $P$. aeruginosa in 2008 in patients after ERCP in the UMCG (Kovaleva et al., 2009). Recently in France hospitals duodenoscopes-related nosocomial infections due to Klebsiella pneumoniae producing extended-spectrum betalactamase (ESBL) occurred (Aumeran et al., 2010) and also of even more multidrugresistant K. pneumoniae carbapenemase (KPC)-producing K. pneumoniae type 2 (Carbonne et al., 2010).

\subsection{Endoscope reprocessing}

Accurate reprocessing of flexible endoscopes must involve cleaning, followed by high level disinfection with further rinsing and drying before storage. If the programme does not include an adequate drying phase, like after manual disinfection, the instrument must be disinfected again if it has not been in use for longer than 4 hours (Dutch Infection Prevention Working Party, 2004). Because almost all outbreaks are related to breaches in reprocessing techniques, it is crucial that endoscope cleaning and disinfection are performed carefully. However, process control of the endoscope reprocessing does not guarantee prevention of settlement of biofilm and consequent shedding of bacteria during endoscopy. Disinfection and drying procedures may fail in the endoscope with a manufacturing defect or damaged internal channels when the decontamination process cannot reach all internal parts of the instrument, and biofilm formation will occur within the endoscope channels (Kovaleva et al., 2009; Srinivasan, 2003).

At the UMCG endoscope reprocessing is performed according to the recommendations of the Dutch national guideline (Dutch Infection Prevention Working Party, 2004) and to the manufacture's recommendations. After manual pre-cleaning and cleaning with detergent (Neodisher Mediclean, Dr. Weigert, Hamburg, Germany) and rinsing with water, endoscopes are disinfected 10 minutes at the temperature of $25^{\circ} \mathrm{C}$ in one of the automatic washer disinfectors (WD 440, Wassenburg Medical Devices B.V., The Netherlands) with $1 \%$ Neodisher Septo PAC (Dr. Weigert, Hamburg, Germany). This disinfectant contains a blend of peracetic acid $(1 \%)$, acetic acid $(<20 \%)$ and hydrogen peroxide (15-30\%). After disinfection endoscopes are dried for 2 hours at $50{ }^{\circ} \mathrm{C}$ using sterile compressed air and hereafter are stored in drying cabinets with continuous flow of dry compressed air at room 
temperature. If a flexible endoscope is used for different patients in a single day, the disinfection and drying procedures will be performed between patients.

\subsection{Surveillance algorithm}

In the UMCG the surveillance system for evaluation of the efficacy of the endoscope reprocessing was implemented. According to our surveillance protocol, therapeutic gastro- and duodenoscopes are microbiologically tested monthly and diagnostic endoscopes once every 3 months (Buss et al., 2008; Kovaleva et al., 2009). A decision algorithm was designed for interpretation of the relevance of positive culture results (Figure 1).

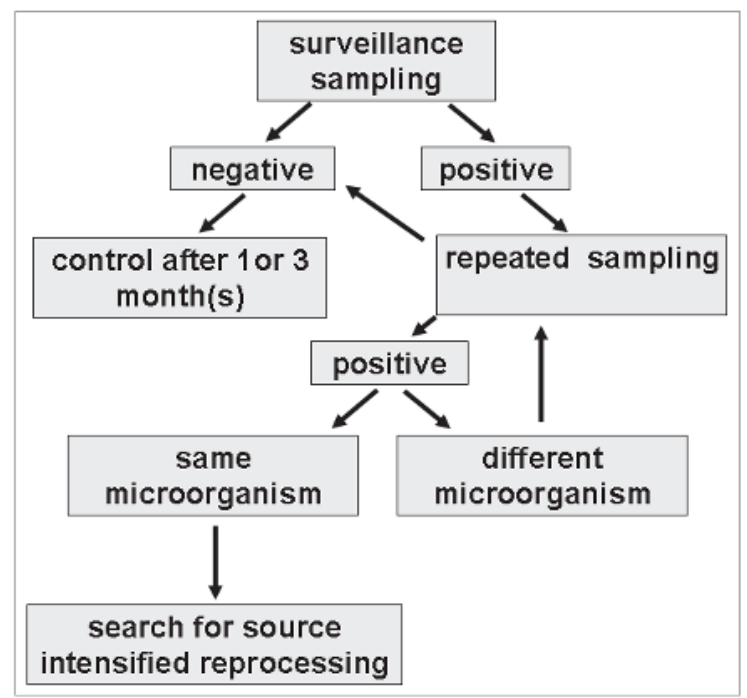

Fig. 1. Decision algorithm for endoscope surveillance.

If, in terms of the decision algorithm, a contamination problem occurs or clinical data suggest transmission, additional samples are taken from the washer disinfectors and also from the tubes connecting the washer disinfectors and the endoscopes and other endoscope accessories. Culturing of special microorganism (e.g. mycobacteria) might be indicated. After a single contamination with a relevant microorganisms (e.g., Enterobacteriaceae, Candida species, gram-negative nonfermenters, Staphylococcus aureus), the endoscope is taken out of service in patients and the results of repeat sampling are awaited. Such endoscopes undergo an intensive high level disinfection procedure. Endoscopes with positive cultures for the same clinically relevant microbial species in two consecutive tests after high level disinfection procedures stay out of use and undergo an additional reprocessing step using sterilization with ethylene oxide followed by microbiological testing before use.

A contaminated endoscope should be inspected for potential defects. Unfortunately, it is not possible to adequately check all internal endoscope channels. Cracking, splitting, channel wall holes can all be the source of bacterial contamination within the endoscope which can be difficult to impossible to detect by routine inspection and testing. 
In the literature there are no standards about frequency of the testing intervals of surveillance cultures. The Gastroenterological Society of Australia guideline (2006) recommends the microbiologic monitoring of ERCP endoscopes and bronchoscopes every 4 weeks and all other gastrointestinal endoscopes every 4 months. According to the European Society of Gastrointestinal Endoscopy guideline, routine microbiological testing of endoscopes is advised to perform at intervals no longer than 3 months (Beilenhoff et al., 2007).

Every microbiological surveillance system has one important limitation. If there is a clinical demand for reuse of an endoscope in the mean time, surveillance culture results will likely not be obtained until after the endoscope is used on the next patient because cultures take a minimum of 24 to 48 hours to incubate (Beilenhoff et al., 2007).

\subsection{Sampling technique}

Sampling of patient-ready endoscopes can be performed with two different techniques. For anterograde sampling, the last rinse water from the endoscope is collected inside the washer disinfector at the distal end of the endoscope. At the endoscopy center in the UMCG, we use a retrograde technique to obtain surveillance specimens for culture from the biopsy/suction and the water/air endoscope channels (Buss et al., 2008; Kovaleva et al., 2009).

For retrograde sampling, the biopsy/suction channel and the water/air channel of the endoscope are each flushed with $20 \mathrm{ml}$ sterile demineralised water manually outside the washer disinfector from the distal to the proximal end. To allow the water to enter the small distal openings of the channels, we connect the syringe with an adapter taken from a gastrointestinal dilator (Savary-Gilliard Dilators; Cook Medical, Bloomington, IN, USA). For sampling under sterile conditions this work has to be done by two persons. The water is collected at the proximal ends of the channels. The biopsy/suction channel is flushed a second time after collecting the water at the suction port and the endoscope is then turned upside-down in order to collect the fluid at the biopsy port. Sampling options can differ depending on the design of the endoscope and the washer disinfector. Samples should be processed shortly after collecting. All samples from endoscopes are sent for culturing. In case of positive results all isolated strains are stored.

If sampling of washer disinfectors is indicated samples from all water channels, the water inlet and the water reservoir and from the tubes connecting the washer disinfectors and the endoscopes should be taken. To enhance the sensitivity of culturing, the washer disinfector can be taken out of use at the end of a normal working day and samples should be taken several days later or at least after one overnight storage period. Following this procedure an auto-disinfection cycle can be run and one to several days later a second sampling can be performed.

The endoscopes have to be sampled in a retrograde manner, because anterograde sampling is not sensitive enough, as shown by our own data (Buss et al., 2008) and those of another study (Srinivasan et al., 2003). Srinivasan et al. (2003) and Cêtre et al. (2005) sampled the biopsy suction channel retrogradely using the suction button of bronchoscopes to suck back the sample fluid, used for flushing, to the proximal channel opening. In our approach the water and air channels of gastrointestinal endoscopes were sampled retrogradely. Alternatively, flushing through combined with brushing may be used (Rejchrt et al., 2004). During the endoscopy-related outbreak caused by ESBL-positive K. pneumoniae in France the epidemic strain was finally isolated from one duodenoscope by flushing and brushing the channels (Aumeran et al., 2010). We know no comparison of the sensitivities of these variants with that of our way of retrograde sampling. The mechanisms underlying the 
higher sensitivity of retrograde sampling need to be further investigated. Probably, changing the direction of the water flow leads to easier removal of organic material and biofilm that have grown with the anterograde flow of the disinfection procedure. Niches inside the endoscopes that are not reached by the anterograde sampling flow may become more accessible.

\subsection{Laboratory protocol}

One hundred $\mathrm{mL}$ of each sample are inoculated on agar plates for aerobic culturing as follows: blood agar (BA; 5\% blood sheep agar; Oxoid Ltd., Cambridge, UK), chocolate blood agar (CHOC; Oxoid), Sabouraud dextrose agar with $0.02 \mathrm{~g} / \mathrm{L}$ aztreonam and $0.02 \mathrm{~g} / \mathrm{L}$ vancomycin (SABav; Oxoid), and tryptone glucose extract agar (TGE; Oxoid). The cultures are incubated for 4 days at $35^{\circ} \mathrm{C}$ (BA, CHOC, SABav) or 7 days at room temperature (TGE). In addition, $1 \mathrm{~mL}$ of each sample are added to an aerobic blood culture bottle and incubated for 4 days in the BacT/ALERT system (bioMérieux SA, Marcy l'Etoile, France). Alternatively to the use of a broth medium, samples can be filtrated to concentrate the microorganisms and the filters cultured.

Bacterial isolates and yeasts are identified according to standard determination schemes. If indicated, culturing of mycobacteria can be done following standard sampling procedures. We do not culture for anaerobic microorganisms nor do we carry out virus detection.

\subsection{Culture results and interpretation}

In 2008208 retrograde samples from endoscopes were taken. With retrograde sampling, $20 \%(42 / 208)$ of all endoscope cultures were found to be contaminated with a microorganism; $5.3 \%(11 / 208)$ of the tests resulted in withdrawal of the contaminated endoscopes, followed by repeated intensified disinfection procedures and gas sterilization. Previous surveillance data from September 2002 until January 2005 demonstrate the much higher sensitivity of retrograde versus anterograde taken samples. The percentage of positive cultures of anterograde versus retrograde samples were $7.9 \%$ respectively $31 \%$ for all microorganisms and for yeasts alone $2.8 \%$ respectively $25 \%$ (Buss et al., 2008).

Various groups of bacteria were found in the surveillance cultures. The following microorganisms were frequently found: aerobic gram-positive cocci, Enterobacteriaceae, gram-negative nonfermenters (Pseudomonas, Acinetobacter species). Repeated endoscope contamination with Stenotrophomonas maltophilia and Methylobacterium is regularly demonstrated, after which intervention conform the protocol is implemented. Frequently more than one species was found in one culture; in many cases this was a mix of Candida species with gram-negative rods or nonfermenters.

In the course of time we found an increasing number of endoscopes positive for nonalbicans Candida species, in particular Candida parapsilosis $(77 \%$ of all further identified non-albicans Candida species). The persistent culturing of C. parapsilosis was generally an indicator of a disinfection problem and may be caused by a low disinfection temperature.

\subsection{Economic burden of endoscope reprocessing and endoscopy-related infection}

The literature related to the costs associated with endoscope reprocessing and the economic consequences of endoscope contamination is very limited. The economic evaluation of 
exogenous endoscopy-related outbreaks should include the analysis of outcomes (the number of prevented exogenous endoscopy-related infections) and the estimation of the health care, non-health care and indirect costs (Seoane-Vazquez et al., 2006). The health care costs include the costs of the epidemiological investigation of the outbreak, the diagnosis and treatment of the affected patients, and the use of resources cost associated with endoscope unit inactivity. A study of post-arthroscopy infections estimated the cost approximately at $\$ 9,155$ per case (Babcock et al., 2003).

The economic evaluation of the microbiological surveillance should include a calculation of the costs for the microbiological tests, costs in time for nursing staff to collect the samples, costs of endoscope recall and extra disinfection procedures or service of the endoscopes. Recent articles pointed to the high cost of periodic monitoring of endoscopes, washer disinfectors and the environment. A recently published study analysed the cost of 4-weekly surveillance microbiological tests of bronchoscopes, duodenoscopes and automated washer disinfectors during a 5-year period. The overall cost of performed tests and cost in time for nursing staff to collect the samples was estimated at \$AUD 100.400 (Gillespie et al., 2008). The costs of an outbreak with a number of clinical complications as described in our cases are usually a multiple of this amount. Microbiological surveillance of endoscope reprocessing and intervention protocol can save the high costs associated with incidental cases and outbreaks of endoscopy-related infections.

In the economic analyses performed in 2008 in the UMCG, the costs of the surveillance of the endoscope reprocessing (cost of tests and cost in time for staff) were estimated at $€$ 25.967. Not calculated were the costs for reprocessing and/or revision of endoscopes by the manufacturer. It was estimated that the costs of the recent Pseudomonas outbreak, i.e. the direct health-care costs for the diagnosis, treatment and hospitalization of the six affecting patients, approximated $€ 157.729$.

\subsection{Summary and future prospects}

The UMCG has 9 years of experience with the microbiological monitoring of flexible endoscopes, biofilm detection and intervention. Positive results of microbiological surveillance have been leading to withdrawal, additional disinfection and incidental gas sterilization of the contaminated endoscopes. The risk of contamination and infection were reduced for large groups of patients, and the expected costs of the post-endoscopic infectious complications were saved (Buss et al, 2008; Kovaleva et al., 2009). However, our surveillance and intervention did not prevent six serious infections by the genotypic similar strain of $P$. aeruginosa, due to the window period in between the surveillance procedures ( 1 month).

Our protocol is able to detect a structural problem in the endoscope disinfection process. Retrograde sampling is crucial for this purpose, because it has much higher sensitivity than anterograde sampling (Buss et al., 2008; Srinivasan et al., 2003). Endoscopes with damaged working channels often are the source of the contamination problems.

Our monitoring and decision algorithm is a product of a learning-by-doing process when we were confronted with contamination problems. If we had not done retrograde sampling we would not have recognized the structural disinfection problems of individual endoscopes. Ideally, an easily performed daily microbiological surveillance method will be developed in the future, which can replace culturing and give faster results. This technique might be based on adenosine triphosphate measuring or polymerase chain reaction detection (Obee et al., 2005; Sciortino et al., 2004). 
Some investigators have begun exploring sterile-sheathed endoscopes with new technology to reduce the risk for infectious complications. The EndoSheath is an endoscope system in which all parts of the endoscope that come into contact with the patient are fully disposable (Colt et al., 2000; Srinivasan, 2003). The endoscope does not come in contact with the patient and does not require disinfection. The disposable sheath, which is in contact with the patient, includes the air/water, suction, and working channels. The main purposes of the sheath are to prevent the transmission of pathogens, and to reduce the need for high-level sterilization.

\section{Impact of biofilms on endoscope reprocessing}

\subsection{Definition and properties of biofilm}

Microorganisms in nature do not generally grow in nutrient-rich suspensions as in the laboratory, but prefer to grow in surface-associated communities, called biofilms (Bar-Or, 1990). The phenomenon of biofilm formation by microorganisms on inert surfaces has been extensively studied and there appears to be a direct relationship between the ability of the organism to form a biofilm and its pathogenicity (Shin et al., 2002).

A biofilm is an assemblage of microbial cells that is irreversibly attached to a surface and enclosed in an exopolysaccharide matrix (Donlan, 2002). Biofilms consist of a substratum to which the microorganism adheres, a conditioning film, the matrix and the liquid phase (Costerton et al., 1995). A typical biofilm will contain around $85 \%$ polysaccharide matrix and only $15 \%$ bacterial mass. Biofilms may form on different surfaces, including living or dead tissues, indwelling medical devices, water supply systems, or endoscope channels (Donlan, 2002; Pajkos et al., 2004).

Bacteria growing within biofilms have a number of characteristics that distinguish them from planktonic populations. Ability to form biofilms allows microorganisms to survive under conditions of drying, chemical and antibiotic exposure. Cells growing within biofilms are protected from host immune system and are frequently 1000 times more resistant to antibiotics than the non-attached planktonic cells. The increased resistance to antimicrobial agents can be explained by poor penetration of an antibiotic into a biofilm, slow growth rate and formation of resistant phenotypes of microorganisms within biofilms (Costerton et al., 1995; Patel, 2005).

Under adverse conditions, biofilms are capable to release their bacterial population into a planktonic state. This ability can be explained by intercellular communication within a biofilm (Davies et al., 2008). Signalling systems include quorum sensing (the release of signal chemicals in response to increasing population density), biosignal blockers, pheromones and butyrylhomoserine lactone. These signalling systems are important in regulation of a number of physiological processes, including antibiotic synthesis, plasmid transfer and the expression of virulence factors (van Delden \& Iglewski, 1998).

With the increasing use of invasive medical procedures, infections involving biofilms form an important risk factor for infectious complications. It has been reported that bacterial biofilm causes about $65 \%$ of bacterial infections in the clinic (Chicurel, 2000). These infections often develop in immunocompromised patients and can be caused by a variety of different microorganisms. Biofilm infections show poor manifestation and recurring symptoms after antibiotic therapy (Costerton et al., 1995). Bacteria within biofilms are able to evade the host immune system. Antibodies are not effective against microorganisms within biofilms, and immune complex can damage the surrounding tissues (Cochrane et al., 1985). 


\subsection{Removal of biofilms}

During endoscopy, the environment provides optimal conditions for the growth of biofilms. Modern endoscopes contain multiple channels and ports which allow for the collection of organic material. The presence of biofilms on the inner surface of endoscope channels has been reported in many publications (Buss et al., 2008; Kovaleva et al., 2009; Pajkos et al., 2004). Even if valid endoscope reprocessing protocols are applied, microbial accumulation can lead to the development of a mature biofilm inside narrow endoscope channels in time (Pajkos et al., 2004). Biofilm formation on the inner surface of the endoscope channels, especially, when these become scratched or damaged, can result in failure of the decontamination process. It can create a vicious circle of growth, disinfection, partial killing or inhibition and regrowth, resulting in outbreaks of endoscopy-related infections in patients who underwent an endoscopy with the biofilm-containing endoscope (Buss et al., 2008; Kovaleva et al., 2009).

Scanning electron microscopy is able to reveal incomplete removal of debris from the interior part of a dismantled endoscope after reprocessing. Figure 2 shows a bacterial biofilm inside an endoscope channel. This endoscope was contaminated not only the inside but also the outside of the channels.

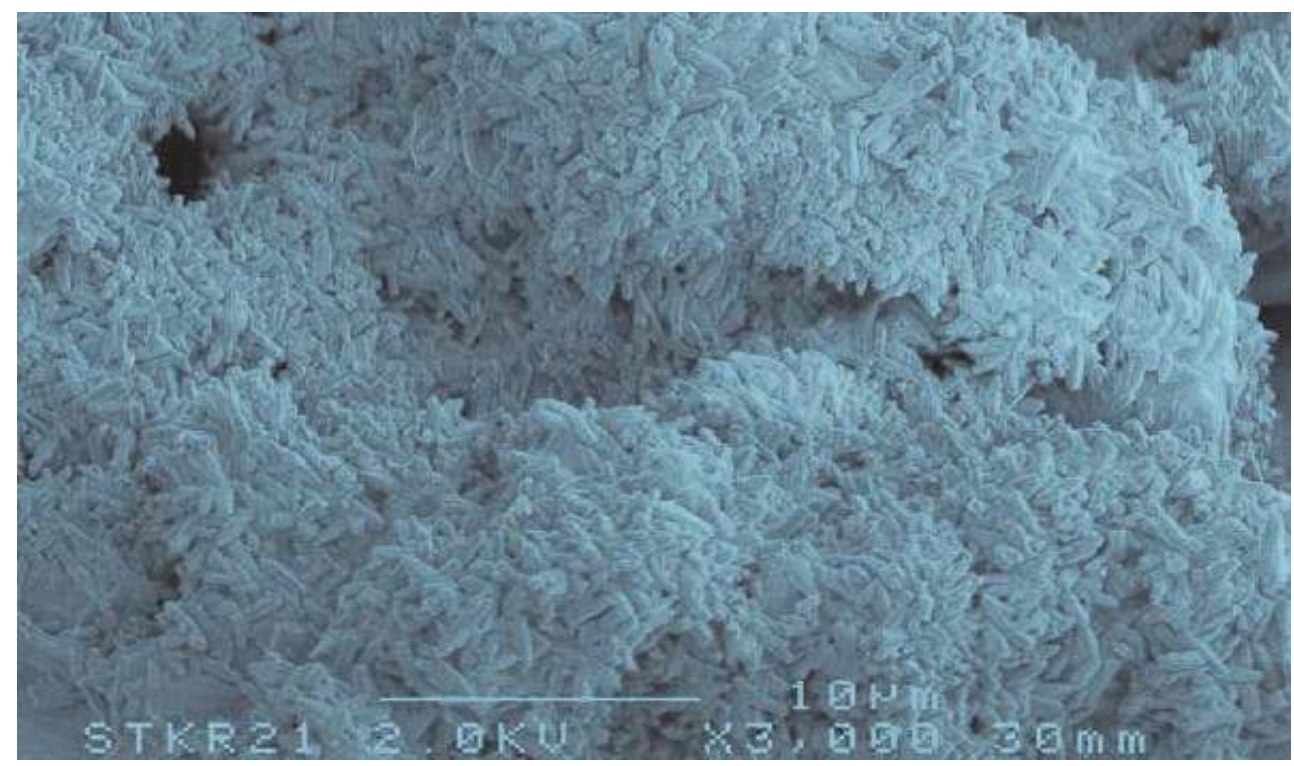

Fig. 2. Scanning electron microscopy picture of bacterial biofilm in an endoscope channel.

Oxidising agents are the most effective chemicals currently available for killing and removing biofilms. Peracetic acid is an oxidising agent usually used for high-level disinfection of flexible endoscopes. It is effective against bacteria, fungi, spores and viruses, minimally toxic and not corrosive at the concentration recommended for disinfection. The biocidal effect of peracetic acid on sessile microorganisms is well known (Henoun Loukili et al., 2004). However, the effect of this disinfectant on biological 
deposits, particularly its ability to fix or to remove biofilms from materials, is not completely studied.

To have potential action on biofilms, relatively high concentrations of the disinfecting agent are necessary. Current peracetic acid disinfectants are not used in high concentrations for endoscope reprocessing because of corrosive properties (Gastroenterological Society of Australia, 2006). According to Marion-Ferey et al. (2003), peracetic acid had a limited efficacy in biofilm removal from the silicone tubing in haemodialysis systems. Other studies demonstrated the ability of peracetic acid disinfectants to fix the E. coli biofilm and blood on to artificial materials (Henoun Loukili et al., 2004; Kampf et al., 2004)

Glutaraldehyde is also an effective agent in endoscope disinfection. It requires a much greater time to kill the biofilm and is less effective in causing shedding of the biofilm from the surface. However, glutaraldehyde is irritating to the skin, can cause allergic contact dermatitis, and may result in irritation of the eyes and nasal mucosa (Alvarado \& Reichelderfer, 2000). Recently, resistance to glutaraldehyde against some $M$. chelonae strains has been described in the literature (van Klingeren \& Pullen, 1993). Kovacs et al. (1998) reported a strain of $P$. aeruginosa responsible for three separate clinical episodes of ERCPassociated cholangitis and concluded that the organism developed adaptive chemical resistance to glutaraldehyde.

The use of anti-biofilm oxidising agents and disinfectants with antimicrobial coating inside washer disinfectors could reduce biofilm build-up inside endoscopes and endoscope washers and decrease the risk of transmitting infections (Marion et al., 2006; Rutala \& Weber, 2001).

Sterilization can be helpful to destroy microorganisms within biofilms. However, flexible endoscopes cannot be heat-sterilized because of vulnerable elements. Low temperature hydrogen peroxide plasma and ethylene oxide gas sterilization can be used during endoscope reprocessing. Recent studies indicate problems with gas and plasma sterilization of long, narrow lumina in the presence of organic soil and salts (Gastroenterological Society of Australia, 2006).

\subsection{Role of drying in biofilm removal}

Accurate drying and storage are important factors in the maintenance of bacteria-free endoscopes. The potential for microbial growth inside endoscope channels after disinfection mainly depends on the conditions within the endoscope channels during drying and storage. Wet channels after reprocessing facilitate growth of such microorganisms as Pseudomonas and Acinetobacter species during storage (Alfa \& Sitter, 1991). The drying procedure of the endoscopes after cleaning at $50{ }^{\circ} \mathrm{C}$, followed by drying with a constant flow of compressed ambient air is applied in the UMCG.

According to the literature, endoscopes stay bacteria-free after prolonged storage if an adequate drying procedure was applied. When stored in the drying cabinet with a laminar air flow, no growth of bacteria and Candida species was found in endoscopes channels 5 days after reprocessing (Rejchrt et al., 2004). Allen et al. (1987) pointed the high efficiency of a drying procedure with suctioning $70 \%$ alcohol through endoscope channels, followed by compressed air in prevention of the further contaminations during the $P$. aeruginosa infection outbreak.

We recently demonstrated that routine cleaning procedures can not always remove biofilm reliably from endoscope channels if the accurate drying procedure is not applied. We 
studied the ability for biofilm formation and the effects of the peracetic acid-based disinfectant, currently in use for endoscope reprocessing in the UMCG, with, and without additional drying on Candida albicans, C. parapsilosis, P. aeruginosa and S. maltophilia biofilms (Kovaleva et al., 2010).

The biofilms were prepared in sterile tissue culture polystyrene 96-well microtiter plates. After $72 \mathrm{~h}$ incubation, single- and dual-species biofilms were treated for $10 \mathrm{~min}$ with the disinfectant at concentrations conform the minimal bactericidal concentration of the strains tested and at $1 \%$ concentration, recommended for disinfection of flexible endoscopes by the company. In order to mimic the biofilm formation and to establish a possible regrowth of biofilms inside the endoscope channels after reprocessing, we developed an in vitro biofilm model which underwent the different steps of the disinfection and drying procedures applied for reprocessing of flexible endoscopes (Figure 3). The viability of the biofilm was quantified by using the tetrazolium salt (MTT) reduction assay and by counting colony-forming yeasts and bacteria of the 10 -fold serial biofilm dilutions on agar plates.

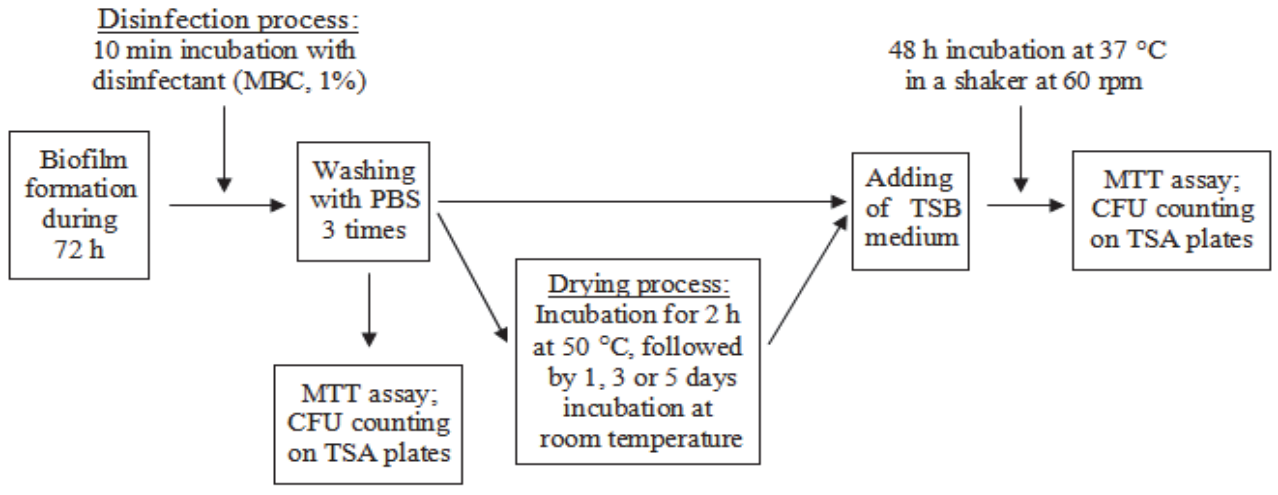

Fig. 3. In vitro biofilm model mimicking the different steps of the disinfection procedure without and with the drying process in a flexible endoscope. CFU, colony-forming units; MTT, tetrazolium salt reduction assay; MBC, minimal bactericidal concentration; TSA, trypticase soy agar; PBS, phosphate buffered saline.

A low MTT formazan signal was demonstrated in all biofilms directly after $10 \mathrm{~min}$ treatment with the minimal bactericidal concentration and $1 \%$ disinfectant (Figure $4 \mathrm{a}$ ). A total inhibition of microbial growth in all biofilms on agar plates occurred after treatment with $1 \%$ disinfectant. Minimal bactericidal concentration caused a marked inhibition of microbial growth of all biofilms but not a $100 \%$ bactericidal and fungicidal efficacy. Regrowth of all biofilms occurred following $48 \mathrm{~h}$ incubation with trypticase soy broth directly after treatment with the minimal bactericidal concentration and $1 \%$ disinfectant if the drying procedure was skipped. No biofilm regrowth occurred in wells after a drying procedure for 1, 3 and 5 days in all biofilms (Figure $4 b$ ). 


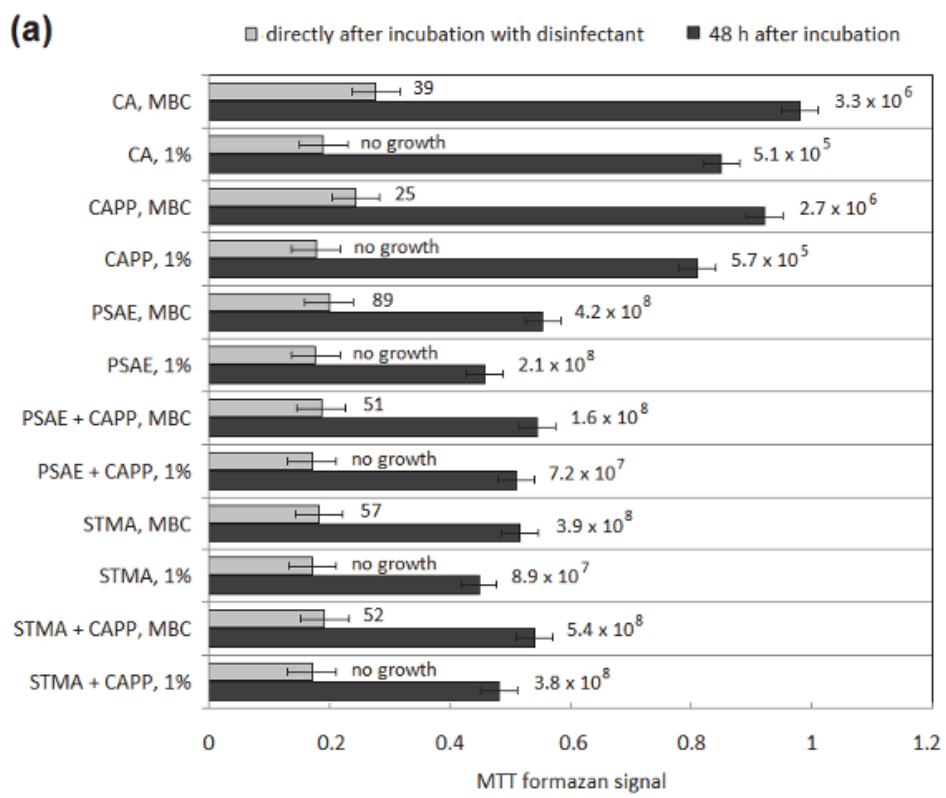

(b)

Fig. 4. Regrowth of the single- and dual-species C. albicans (CA), C. parapsilosis (CAPP), P. aeruginosa (PSAE) and S. maltophilia (STMA) biofilms expressed in vitality by measuring the MTT signal and expressed in viability in CFU per well after the disinfection procedure with MBC and 1\% disinfectant (a) without additional drying and (b) with additional drying for 1 , 3 and 5 days. 
This study demonstrated the high efficacy of the drying procedure after the disinfection step against yeasts and bacteria in all single- and dual-species biofilms. Failure of decontamination endoscopes can be explained by an invalid drying procedure.

\section{Conclusion}

Contaminated endoscopes have been linked to many outbreaks of device-related nosocomial infections. The true incidence of endoscopy-related infections is unknown because of inadequate surveillance or no surveillance at all. Endoscopy-related infections can cause serious harm and can give rise to concerns over these procedures by physicians and patients in.

Flexible endoscopes can be cleaned and disinfected but not sterilized after use. This implies the risk of settlement of biofilm producing species. Routine cleaning and disinfection procedures do not remove biofilm reliably from endoscope channels if the accurate drying procedure is not applied. This may explain the failure of decontamination during endoscope reprocessing.

Process control of the washing and disinfection procedure is requested and implemented but does not guarantee prevention of settlement of biofilm and consequent shedding of bacteria during endoscopy. Implementation of a microbiological surveillance of endoscope reprocessing is appropriate to detect early colonization and biofilm formation in the endoscope and to prevent contamination and infection in patients after endoscopic procedures. However, the returns of prevention of endoscopy-related infections should be reasonably in balance with the costs of the technical and laboratory procedures resulting from surveillance and the costs of reprocessing or servicing of the contaminated endoscope.

\section{Acknowledgment}

The authors wish to thank Prof. Dr. John Degener and Prof. Dr. Henny van der Mei for their advice and support. We thank the staff of the Departments of Medical Microbiology and Endoscopy of the UMCG for technical assistance and collaboration.

\section{References}

Allen, J.; Allen, M.; Olson, M.; Gerding, D.; Shanholtzer, C.; Meier, P.; Vennes, J. \& Silvis, S. Pseudomonas Infection of the Biliary System Resulting from Use of a Contaminated Endoscope. Gastroenterology, Vol.92, No.3, (March 1987), pp. 759-763, ISSN 00165085

Alfa, M. \& Sitter, D. In-hospital Evaluation of Contamination of Duodenoscopes: a Quantitative Assessment of the Effect of Drying. Journal of Hospital Infection, Vol.19, No.2, (October 1991), pp. 89-98, ISSN 0195-6701

Alvarado, C. \& Reichelderfer, M. APIC Guidelines for Infection Prevention and Control in Flexible Endoscopy. American Journal of Infection Control, Vol.28, No.2, (April 2000), pp. 138-155, ISSN 0196-6553

Alvarado, C.; Stolz, S. \& Maki, D. Nosocomial Infections from Contaminated Endoscopes: a Flawed Automated Endoscope Washer. An Investigation Using Molecular Epidemiology. The American Journal of Medicine, Vol. 91, No.3B, (September 1991), pp. 272-280, ISSN 0002-9343 
Anderson, D.; Shimpi, R.; McDonald, J.; Branch, M.; Kanafani, Z.; Harger, J.; Ely, T.; Sexton, D. \& Kaye, K. Infectious Complications Following Endoscopic Retrograde Cholangiopancreatography: an Automated Surveillance System for Detecting Postprocedure Bacteraemia. American Journal of Infection Control, Vol.36, No.8, (October 2008), pp. 592-594, ISSN 0196-6553

Aumeran, C.; Poincloux, L.; Souweine, B.; Robin, F.; Laurichesse, H.; Baud, O.; Bommelaer, G. \& Traoré, O. Multidrug-resistant Klebsiella Pneumoniae Outbreak after Endoscopic Retrograde Cholangiopancreatography. Endoscopy, Vol.42, No. 11, (November 2010), pp. 895-899, ISSN 0013-726X

Babcock, H.; Carroll, C.; Matava, M.; L'ecuyer, P. \& Fraser, V. Surgical site infections after arthroscopy: outbreak investigation and case control study. Arthroscopy: The Journal of Arthroscopic and Related Surgery, Vol.19, No.2, (February 2003), pp. 172-181, ISSN 0749-8063

Bar-Or, Y. The Effects of Adhesion on Survival and Growth of Microorganisms. Experientia, Vol.46, No.8, (1990), pp. 823-826, ISSN 0014-4754

Bass, D.; Oliver, S. \& Bornman, P. Pseudomonas Septicaemia after Endoscopic Retrograde Cholangiopancreatography - an Unresolved Problem. South African Medical Journal, Vol.77, No. 10, (May 1990), pp. 509-511, ISSN 0256-9574

Beilenhoff, U.; Neumann, C.; Rey, J.; Biering, H.; Blum, R.; Schmidt, V. \& ESGE Guidelines Committee. ESGE-ESGENA Guideline for Quality Assurance in Reprocessing: Microbiological Surveillance Testing in Endoscopy. Endoscopy, Vol.39, No.2, (February 2007), pp. 175-181, ISSN 0013-726X

Bilbao, M.; Dotter, C.; Lee, T. \& Katon, R. Complications of Endoscopic Retrograde Cholangiopancreatography (ERCP). Gastroenterology, Vol. 70, No.3, (March 1976), pp. 314-320, ISSN 0016-5085

Bryce, E.; Walker, M.; Bevan, C. \& Smith J. Contamination of Bronchoscopes with Mycobacterium Tuberculosis. Canadian Journal of Infection Control, Vol.8, No.2, (Summer 1993), pp. 35-36, ISSN 1183-5702

Buss, A.; Been, M.; Borgers, R.; Stokroos, I.; Melchers, W.; Peters, F.; Limburg, A. \& Degener, J. Endoscope Disinfection and its Pitfalls - Requirement for Retrograde Surveillance Cultures. Endoscopy, Vol.40, No.4, (February 2008), pp. 327-332, ISSN 0013-726X

Carbonne, A.; Thiolet, J.; Fournier, S.; Fortineau, N.; Kassis-Chikhani, N.; Boytchev, I.; Aggoune, M.; Séguier, J.; Sénéchal, H.; Tavolacci, M.; Coignard, B.; Astagneau, P. \& Jarlier, V. Control of a Multi-hospital Outbreak of KPC-producing Klebsiella Pneumoniae Type 2 in France, September to October 2009. Eurosurveillance, Vol.48, No.15, (December 2010), pii: 19734, ISSN 1560-7917

Cêtre, J.-C.; Nicolle, M.-C.; Salord, H.; Pérol, M.; Tigaud, S.; David, G.; Bourjault, M. \& Vanhems, P. Outbreaks of Contaminated Broncho-alveolar Lavage Related to Intrinsically Defective Bronchoscopes. Journal of Hospital Infection, Vol.61, No.1, (September 2005), pp. 39-45, ISSN 0195-6701

Chicurel, M. Bacterial Biofilms and Infections. Slimebusters. Nature, Vol.408, No.6810, (November 2000), pp. 284-286. ISSN 0028-0838

Christensen, M.; Matzen, P.; Schulze, S. \& Rosenberg, J. Complications of ERCP: a Prospective Study. Gastrointestinal Endoscopy, Vol.60, No.5, (November 2004), pp. 721-731, ISSN 0016-5107 
Classen, D.; Jacobson, J.; Burke, J.; Jacobson, J. \& Evans, R. Serious Pseudomonas Infections Associated with Endoscopic Retrograde Cholangiopancreatography. The American Journal of Medicine, Vol.84, No.3, (March 1988), pp. 590-596, ISSN 0002-9343

Cochrane, D.; Brown, M.; Anwar, H.; Weller, P.; Lam, K. \& Costerton, J. Antibody Response to Pseudomonas Aeruginosa Surface Protein Antigens in a Rat Model of Chronic Lung Infection. Journal of Medical Microbiology, Vol.27, No.4, (December 1988), pp. 255-261.

Colt, H.; Beamis, J.; Harrell, J. \& Mathur, P. Novel Flexible Bronchoscope and Single-use Disposable-sheath Endoscope System. A Preliminary Technology Evaluation. Chest, Vol.118, No.1, (July 2000), pp. 183-187, ISSN 0012-3692

Corne, P.; Godreuil, S.; Jean-Pierre, H.; Jonquet, O.; Campos, J.; Jumas-Bilak, E.; Parer, S \& Marchandin, H. Unusual Implication of Biopsy Forceps in Outbreaks of Pseudomonas Aeruginosa Infections and Pseudo-infections Related to Bronchoscopy. Journal of Hospital Infection, Vol.61, No.1, (September 2005), pp. 2026, ISSN 0195-6701

Costerton, J.; Lewandowski, Z.; Caldwell, D.; Korber, D \& Lappin-Scott, H. Microbial Biofilms. Annual Reviews in Microbiology, Vol.49, (October 1995), pp. 711-745, ISSN 0066-4227

Costerton, J.; Stewart, P. \& Greenberg, E. Bacterial Biofilms: a Common Cause of Persistent Infections. Science, Vol.284, No.5418, (May 1999), pp. 1318-1322, ISSN 0036-8075

Cotton, P.; Connor, P.; Rawls, E. \& Romagnuolo, J. Infection after ERCP and Antibiotic Prophylaxis: a Sequential Quality-Improvement Approach over 11 Years. Gastrointestinal Endoscopy, Vol.67, No.3, (March 2008), pp. 471-475, ISSN 0016-5107

Cotton, P.; Lehman, G.; Vennes, J.; Geenen, J.; Russell, R.; Meyers, W.; Liguory, C. \& Nickl, N. Endoscopic Sphincterotomy Complications and their Management: an Attempt at Consensus. Gastrointestinal Endoscopy, Vol.37, No.3, (May-June 1991), pp. 383-393, ISSN 0016-5107

Davies, D.; Parsek, M.; Pearson, J.; Iglewski, B.; Costerton, J. \& Greenberg, E. The Involvementof Cell-to-cell Signals in the Development of a Bacterial Biofilm. Science, Vol. 280, No.5361, (April 1998), pp. 295-298, ISSN 0036-8075

Dutch Infection Prevention Working Party (WIP). Guideline for Cleaning and Disinfection of Endoscopes. (April, 2004). Revision: April, 2009. Available at: http://www.wip.nl/UK/; follow links to Documentation - guideline downloads Hospitals - Cleaning, disinfection and sterilization

Fraser, T.; Reiner, S.; Malczynski, M.; Yarnold, P.; Warren, J. \& Noskin, G. Multidrugresistant Pseudomonas Aeruginosa Cholangitis after Endoscopic Retrograde Cholangiopancreatography: Failure of Routine Endoscope Cultures to Prevent an Outbreak. Infection Control and Hospital Epidemiology, Vol. 25, No. 10, (October 2004), pp. 856-859, ISSN 0899-823X

Fraser, V.; Jones, M.; Murray, P.; Medoff, G.; Zhang, Y \& Wallace, R. Contamination of Flexible Fiberoptic Bronchoscopes with Mycobacterium Chelonae Linked to an Automated Bronchoscope Disinfection Machine. The American Review of Respiratory Diseases, Vol.145, No.4, (April 1992), pp. 853-855, ISSN 0003-0805

Gastroenterological Society of Australia. (March, 2006). Infection control in endoscopy. Digestive Health Foundation, Available from:

http://www.gesa.org.au/pdf/booklets/I_Control_2nd_Edition.pdf 
Gillespie, E.; Kotsanas, D. \& Stuart, R. Microbiological monitoring of endoscopes: 5-year review. Journal of Gastroenterology and Hepatology, Vol.23, No.7, (July 2008), pp. 10691074, ISSN 0815-9319

Harris, A.; Chan, C.; Torres-Viera, C.; Hammett, R. \& Carr-Locke, D. Meta-analysis of Antibiotic Prophylaxis in Endoscopic Retrograde Cholangiopancreatography. Endoscopy, Vol.31, No.9, (November 1999), pp. 718-724, ISSN 0013-726X

Henoun Loukili, N.; Becker, H.; Harno, J.; Bientz, M. \& Meunier O. Effect of Peracetic Acid and Aldehyde Disinfectants on Biofilm. Journal of Hospital Infection, Vol.58, No.2, (October 2004), pp. 151-154, ISSN 0195-6701

Hirota, W.; Petersen, K.; Baron, T.; Goldstein, J.; Jacobson, B.; Leighton, J.; Mallery, J.; Waring, J.; Fanelli, R.; Wheeler-Harbough, J. \& Faigel, D. Guidelines for Antibiotic Prophylaxis for GI Endoscopy. Gastrointestinal Endoscopy, Vol.58, No.4, (October 2003), pp. 475-482, ISSN 0016-5107

Imberta, G.; Secciab, Y. \& La Scola, B. Methylobacterium sp. Bacteraemia due to a Contaminated Endoscope. Journal of Hospital Infection, Vol.61, No.3, (November 2005), pp. 268-270, ISSN 0195-6701

Kampf, G.; Blo, R. \& Martiny, H. Surface Fixation of Dried Blood by Glutaraldehyde and Peracetic Acid. Journal of Hospital Infection, Vol.57, No.2, (June 2004), pp. 139-143, ISSN 0195-6701

Kaye, K.; Macone, A. \& Powel H. Catheter Infection Caused by Methylobacterium in Immunocompromised Hosts: Report of Three Cases and Review of the Literature. Clinical Infectious Diseases, Vol.14, No.5, (May 1992), pp. 1010-1014, ISSN 1058-4838

Kirschke, D.; Jones, T.; Craig, A.; Chu, P.; Mayernick, G.; Patel, J. \& Schaffner, W. Pseudomonas Aeruginosa and Serratia Marcescens Contamination Associated with a Manufacturing Defect in Bronchoscopes. New English Journal of Medicine, Vol. 248, No.3, (January 2003), pp. 214-220, ISSN 0028-4793

Kovacs, B.; Aprecio, R.; Kettering, J. \& Chen, E. Efficacy of Various Disinfectants in Killing a Resistant Strain of Pseudomonas Aeruginosa by Comparing Zones of Inhibition: Implications for Endoscopic Equipment Reprocessing. The American Journal of Gastroenterology, Vol.93, No.11, (November 1998), pp. 2057-2059, ISSN 0002-9270

Kovaleva, J.; Meessen, N.; Peters, F.; Been, M.; Arends, J.; Borgers, R. \& Degener, J. Is Bacteriologic Surveillance in Endoscope Reprocessing Stringent Enough? Endoscopy, Vol.41, No.10, (October 2009), pp. 913-916, ISSN 0013-726X

Kovaleva, J.; Degener, J. \& Van der Mei, H. Mimicking Disinfection and Drying of Biofilms in Contaminated Endoscopes. Journal of Hospital Infection, Vol.76, No.4, (December 2010), pp. 345-350, ISSN 0195-6701

Kressel, A. \& Kidd, F. Pseudo-outbreak of Mycobacterium Chelonae and Methylobacterium Caused by Contamination of an Automated Endoscopy Washer. Infection Control and Hospital Epidemiology, Vol. 22, No.7, (July 2001), pp. 414-418, ISSN 0899-823X

Kullman, E.; Borch, K.; Lindstrom, E.; Ansehn, S.; Ihse, I. \& Anderberg, B. Bacteremia Following Diagnostic and Therapeutic ERCP. Gastrointestinal Endoscopy, Vol.38, No.4, (July - Augustus 1992), pp. 444-449, ISSN 0016-5107

Leers, W. Disinfecting Endoscopes: How not to Transmit Mycobacterium Tuberculosis by Bronchoscopy. Canadian Medical Association Journal, Vol.123, No.4, (Augustus 1980), pp. 275-283, ISSN 1488-2329 
Liu, J.; Wu, J.; Chen, H.; Huang, A.; Ko, W. \& Chuang, Y. Methylobacterium Mesophilicum Synovitis in an Alcoholic. Clinical Infectious Diseases, Vol.24, No.5, (May 1997), pp. 1008-1009, ISSN 1058-4838

Low, D.; Mieflikier, A.; Kennedy, J. \& Stiver, H. Infectious Complications of Endoscopic Retrograde Cholangiopancreatography. A Prospective Assessment. Annals of Internal Medicine, Vol.140, No.8, (Augustus 1980), pp. 1076-1077, ISSN 0003-4819

Marion, K.; Freney, J.; Bergeron, E.; Bergeron, J.; Renaud, F. \& Costerton, J. Using an Efficient Biofilm Detaching Agent: an Essential Step for the Improvement of Endoscope Reprocessing Protocols. Journal of Hospital Infection, Vol.64, No.2, (October 2006), pp. 136-142, ISSN 0195-6701

Marion-Ferey, K.; Pasmore, M.; Stoodley, P.; Wilsony, S.; Husson, G. \& Costerton, J. Biofilm Removal from Silicone Tubing: an Assessment of the Efficacy of Dialysis Machine Decontamination Procedures Using an in Vitro Model. Journal of Hospital Infection, Vol.53, No.1, (January 2003), pp. 64-71, ISSN 0195-6701

Masci, E.; Toti, G.; Mariani, A.; Curioni, S.; Lomazzi, A.; Dinelli, M.; Minoli, G.; Crosta, C.; Comin U.; Fertitta, A.; Prada, A.; Rubis Passoni, G. \& Testoni, P. Complications of Diagnostic and Therapeutic ERCP: a Prospective Multicenter Study. The American Journal of Gastroenterology, Vol.96, No.2, (February 2001), pp. 417-423, ISSN 00029270

Nelson, D.; Barkun, A.; Block, K.; Burdick, J.; Ginsberg, G.; Greenwald, D.; Kelsey, P.; Nakao, N.; Slivka, A.; Smith, P. \& Vakil, N. Technology Status Evaluation Report. Transmission of Infection by Gastrointestinal Endoscopy. Gastrointestinal Endoscopy, Vol.54, No.6, (December 2001), pp. 824-828, ISSN 0016-5107

Nelson, D. \& Muscarella, L. Current Issues in Endoscope Reprocessing and Infection Control during Gastrointestinal Endoscopy. World Journal of Gastroenterology, Vol.12, No.25, (July 2006), pp. 3953-3964, ISSN 1007-9327

Obee, P.; Griffith, C.; Cooper, R.; Cooke, R.; Bennion, N. \& Lewis, M. Real-time Monitoring in Managing the Decontamination of Flexible Gastrointestinal Endoscopes. American Journal of Infection Control, Vol.33, No.4, (May 2005), pp. 202-206, ISSN 0196-6553

O'Connor, H.; Hamilton, I.; Lincoln, C.; Maxwell, S. \& Axon, A. Bacteremia with Upper Gastrointestinal Endoscopy - a Reappraisal. Endoscopy, Vol.15, No.1, (January 1983), pp. 21-23

Pajkos, A.; Vickery, K. \& Cossart, Y. Is Biofilm Accumulation on Endoscope Tubing a Contributor to the Failure of Cleaning and Decontamination? Journal of Hospital Infection, Vol.58, No.3, (November 2004), pp. 224-229, ISSN 0195-6701

Pappas, S.; Schaaff, D.; DiCostanzo, M.; King, F. \& Sharp, J. Contamination of Flexible Fiberoptic Bronchoscopes. The American Review of Respiratory Diseases, Vol.127, No.3, (March 1982), pp. 391-392, ISSN 0003-0805

Patel R. Biofilms and Antimicrobial Resistance. Clinical Orthopaedics and Related Research, No.437, (Augustus 2005), pp. 41-47, ISSN 0009-921X

Rejchrt, S.; Čermák, P.; Pavlatová, L.; Míčková, E. \& Bureš J. Bacteriologic Testing of Endoscopes after High-level Disinfection. Gastrointestinal Endoscopy, Vol.60, No.1, (July 2004), pp. 76-78, ISSN 0016-5107

Rutala, W. \& Weber, D. New Disinfection and Sterilization Methods. Emerging Infectious Diseases, Vol.7, No.2, (March - April 2001), pp. 348-353, ISSN 1080-6059 
Sanders, J.; Martin, J.; Hooke, M. \& Hooke, J. Methylobacterium Mesophilicum Infection: Case Report and Literature Review of an Unusual Opportunistic Pathogen. Clinical Infectious Diseases, Vol.30, No.6, (June 2000), pp. 936-938, ISSN 1058-4838

Schelenz, S. \& French, G. An Outbreak of Multidrug-resistant Pseudomonas Aeruginosa Infection Associated with Contamination of Bronchoscopes and an Endoscope Washer-disinfector. Journal of Hospital Infection, Vol.46, No.1, (September 2000), pp. 23-30, ISSN 0195-6701

Sciortino, C.; Xia, E. \& Mozee, A. Assessment of a Novel Approach to Evaluate the Outcome of Endoscope Reprocessing. Infection Control and Hospital Epidemiology, Vol. 25, No.4, (April 2004), pp. 284-290, ISSN 0899-823X

Shimonom, N.; Takuma, T.; Tsuchimochi N.; Shiose, A.; Kanamoto, M.; Uchida, Y.; Morita, S.; Matsumoto, H \& Hayashi, J. An outbreak of Pseudomonas Aeruginosa Infections Following Thoracic Surgeries Occurring via the Contamination of Bronchoscopes and an Automatic Endoscope Reprocessor. Journal of Infection and Chemotherapy, Vol.14, No.6, (December 2008), pp. 418-423, ISSN 1341-321X

Shin, J.; Kee, S.; Shin, M.; Kim, S.; Shin, D.; Lee, S.; Suh, S. \& Ryang, D. Biofilm Production by Isolates of Candida Species Recovered from Nonneutropenic Patients: Comparison of Bloodstream Isolates with Isolates from other Sources. Journal of Clinical Microbiology, Vol.40, No.4, (April 2002), pp. 1244-1248, ISSN 0095-1137

Seoane-Vazquez, E.; Rodriguez-Monguio, R.; Visaria, J. \& Carlson, A. Exogenous Endoscopy-related Infections, Pseudo-infections, and Toxic Reactions: Clinical and Economic Burden. Current Medical Research and Opinion, Vol.22, No.10, (October, 2006), pp. 2007-2021, ISSN 1473-4877

Seoane-Vazquez, E.; Rodriguez-Monguio, R.; Visaria, J. \& Carlson, A. Endoscopy-related Infections and Toxic Reactions: an International Comparison. Endoscopy, Vol.39, No.8, (Augustus 2007), pp. 742-778, ISSN 0013-726X

Spach, D.; Silverstein, F. \& Stamm, W. Transmission of Infection by Gastrointestinal Endoscopy and Bronchoscopy. Annals of Internal Medicine, Vol.118, No.2, (January, 1993), pp. 117-128, ISSN 1539-3704

Srinivasan, A. Epidemiology and Prevention of Infections Related to Endoscopy. Current Infectious Disease Reports, Vol.5, No.6, (December, 2003), pp. 467-472, ISSN 15343146

Srinivasan, A.; Wolfenden, L.; Song, X.; Mackie, K.; Hartsell, T.; Jones, H.; Diette, G.; Orens, J.; Yung, R.; Ross, T.; Merz, W.; Scheel, P.; Haponik, E. \& Perl, T. An Outbreak of Pseudomonas Aeruginosa Infections Associated with Flexible Bronchoscopes. New English Journal of Medicine, Vol.348, No.3, (January 2003), pp. 221-227, ISSN 00284793

Systchenko, R.; Marchetti, B.; Canard, J.; Palazzo, L.; Ponchon, T.; Rey, J.; Sautereau, D. \& French Society of Digestive Endoscopy. Guidelines of the French Society of Digestive Endoscopy: Recommendations for Setting up Cleaning and Disinfection Procedures in Gastrointestinal Endoscopy. Endoscopy, Vol.32, No.10, (October 2000), pp. 807-818, ISSN 0013-726X

Terhaar sive Droste, J.; Craanen, M.; Kolkman, J. \& Mulder, C. Dutch Endoscopic Capacity in the Era of Colorectal Cancer Screening. The Netherlands Journal of Medicine, Vol.64, No.10, (November 2006), pp. 371-373, ISSN 0300-2977 
Van Delden, C. \& Iglewski, B. Cell-to-cell Signalling and Pseudomonas Aeruginosa Infections. Emerging Infectious Diseases, Vol.4, No.4, (October - December 1998), pp. 551-560, ISSN 1080-6059

Van Klingeren, B. \& Pullen, P. Glutaraldehyde Resistant Mycobacteria from Endoscope Washers. Journal of Hospital Infection, Vol.25, No.2, (October 1993), pp. 147-149, ISSN 0195-6701

Wheeler, P.; Lancaster, D. \& Kaiser, A. Bronchopulmonary Cross-colonization and Infection Related to Mycobacterial Contamination of Suction Valves of Bronchoscopes. The Journal of Infectious Diseases, Vol.159, No.5, (May 1989), pp. 954-958, ISSN 0022-1899 


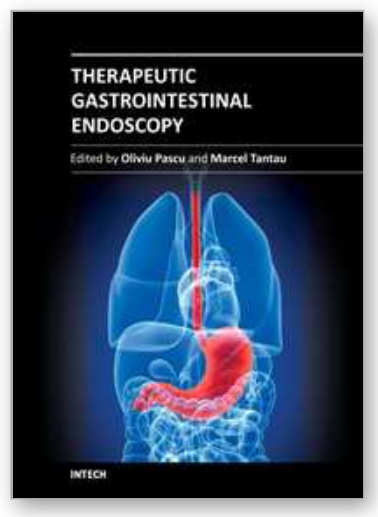

\author{
Therapeutic Gastrointestinal Endoscopy \\ Edited by Prof. Oliviu Pascu
}

ISBN 978-953-307-743-7

Hard cover, 216 pages

Publisher InTech

Published online 22, September, 2011

Published in print edition September, 2011

Endoscopy has had a big role in the development of modern gastroenterology. Modern endoscopy will certainly be more therapeutic. It started with endoscopic hemostasis and polipectomy, than beginning of the 1970 's with the advent of endoscopic sphincterotomy extended to biliopancreatic pathology and has a huge impact in this difficult pathology. Plastic stents made the first steps in endoscopic palliation of neoplastic jaundice, metallic stents, covered or uncovered are better for biliary palliation and can be used also for palliation neoplastic obstruction at different levels of the digestive tube. Resection of digestive tumors has evolved now to sub-mucosal resection, looking to have one-piece complete resection. Interventional endoscopy is now very complex and takes a lot of time for endoscopists to learn properly these techniques. This book is a very good up-to-date overview of new techniques of interventional endoscopy for those who want to learn or develop their knowledges in this field.

\title{
How to reference
}

In order to correctly reference this scholarly work, feel free to copy and paste the following:

Julia Kovaleva and Astrid Buss (2011). Usefulness of Bacteriological Monitoring of Endoscope Reprocessing, Therapeutic Gastrointestinal Endoscopy, Prof. Oliviu Pascu (Ed.), ISBN: 978-953-307-743-7, InTech, Available from: http://www.intechopen.com/books/therapeutic-gastrointestinal-endoscopy/usefulness-of-bacteriologicalmonitoring-of-endoscope-reprocessing 1

\section{INTECH}

open science | open minds

\author{
InTech Europe \\ University Campus STeP Ri \\ Slavka Krautzeka 83/A \\ 51000 Rijeka, Croatia \\ Phone: +385 (51) 770447 \\ Fax: +385 (51) 686166 \\ www.intechopen.com
}

\author{
InTech China \\ Unit 405, Office Block, Hotel Equatorial Shanghai \\ No.65, Yan An Road (West), Shanghai, 200040, China \\ 中国上海市延安西路65号上海国际贵都大饭店办公楼 405 单元 \\ Phone: +86-21-62489820 \\ Fax: $+86-21-62489821$
}


(C) 2011 The Author(s). Licensee IntechOpen. This chapter is distributed under the terms of the Creative Commons Attribution-NonCommercialShareAlike-3.0 License, which permits use, distribution and reproduction for non-commercial purposes, provided the original is properly cited and derivative works building on this content are distributed under the same license. 